\title{
CONFOR PROGRAM: DETERMINATION OF RELATIVE ORIENTATION PARAMETERS BETWEEN VLBI AND FK5 REFERENCE FRAMES
}

\author{
(1) I.I. KUMKOVA, (2) V.V. TEL'NYUK-ADAMCHUK, (2) Yu.G. BABENKO, \\ (2)O.Ya. VERTYPOLOKH \\ (1)--Institute of Applied Astronomy, Russian Academy of Sciences \\ 8 Zhdanovskaya str., \\ 197042 St. Peterburg, Russia \\ (2)--Astronomical Observatory of Kyiv Taras Shevchenko University \\ 3 Observatorna str., \\ 254053 Kyiv, Ukraine
}

\begin{abstract}
The primary goal of the CONFOR Program (CONnection of Frames in Optics and Radio) is to study the connection between VLBI and traditional optical reference frames as well as to study regional features of the FK5 (Gubanov, Kumkova \& Tel'nyuk-Adamchuk, 1990). The main concept of this program is the usage of fixed systems of reference stars in field containing the extragalactic radio sources (ERS) and as a result the creation of a reliable base for astrometric reduction of photographic plates with ERS images. Fulfillment of the Program is embracing several stages.
\end{abstract}

1. Star lists have been prepared of 2575 both intermediate reference stars and radio stars for meridian observations and about 7 thousand stars of 12-14 mag. for astrographic observations in 238 fields with extragalactic compact radio sources.

2. Observations have been organized of these stars with meridian circles and astrographs in several Eastern observatories (Kyiv, Odesa, Bucharest, Mykolaiv, Kharkiv). The meridian observations are finished in Kyiv and Odesa. Positions of radio stars were determined from Kyiv meridian observations (two epochs: 1984 and 1990).

3. Databases of both the optical and radio interferometric observations have been compiled of radio stars, ERS as well as optical observations of intermediate stars in the fields centered in ERS.

4. Observations were carried out with several astrographs (Kyiv, Bucharest, Abastumany, Tautenburg, Kitab, Sanglok) and positions were determined of 57 ERS.

5. Determination was carried out of the angles of relative orientation between VLBI and FK5 reference frames as well as estimation of regional features of the FK5. For this purpose both collected and original radio source coordinates were used.

This work was supported by grant of Academy of Sciences of former Soviet Union and ISF grant U52000.

\section{Reference}

Gubanov, V.S., Kumkova, I.I., and Tel'nyuk-Adamchuk, V.V. (1990) 'CONFOR: a new program for determining the connection between radio and optical reference frames', in J.H. Lieske and V.K. Abalakin (eds.), Inertial Coordinate System on the Sky, Kluwer Academic Publishers, Dordrecht, pp. 75-76. 